\title{
Emprego da nanoargila na remoção de ortofosfato em ensaios de bancada: contribuição para a mitigação do processo de eutrofização
}

\section{Nano clay application in the orthophosphate removal in bench tests: contribution for the mitigation of the eutrophication process}

\author{
Marcio Yukihiro Kohatsu¹, Gabriel Machado², Julio Harada3 ; Tatiane Araujo de Jesus ${ }^{4}$ \\ ${ }^{1}$ Aluno no Curso de Pós-graduação em Ciência e Tecnologia Ambiental, Universidade Federal ABC, Santo \\ André, São Paulo, Brasil. Orcid: https://orcid.org/0000-0003-2998-8780. E-mail: mykohatsu@gmail.com \\ 2Doutor em Engenharia Mineral, Universidade de São Paulo, São Paulo, São Paulo, Brasil. \\ Orcid: https://orcid.org/0000-0001-5351-162X E-mail: gabo.machado@usp.br \\ ${ }^{3}$ Doutor em Nanociências e Materiais Avançados, Universidade Federal do ABC, Santo André, São Paulo, \\ Brasil. Orcid: https://orcid.org/0000-0001-9834-241X. E-mail: harada.julio@terra.com.br \\ ${ }^{4}$ Professora no Programa de Mestrado Acadêmico em Ciência e Tecnologia Ambiental, Universidade \\ Federal do ABC, Santo André, São Paulo, Brasil. Orcid: https://orcid.org/0000-0002-5206-6584. \\ E-mail: tatiane.jesus@ufabc.edu.br
}

\begin{abstract}
RESUMO: Estima-se que, até 2058, pelo menos 55\% dos corpos d'água da América do Sul estarão eutrofizados. Este fenômeno é desencadeado pelo enriquecimento das águas com nutrientes (nitrogênio e fósforo) e acarreta na produção exagerada de algas e plantas aquáticas, redução dos níveis de oxigênio dissolvido e, como consequência, aumento dos custos do tratamento das águas. O fósforo $(\mathrm{P})$, em especial, vem sendo apontado como o principal elemento para o controle da eutrofização. Embora existam tecnologias para remoção de fósforo in situ (ex.: Phoslock ${ }^{\circledR}$ ) sua aplicação em larga escala costuma ser bastante onerosa. Uma alternativa é a nanoargila, que tem baixo custo, é considerada inerte e possui alta reatividade com o fósforo. Assim, o presente estudo teve como objetivo avaliar a eficiência de remoção de ortofosfato utilizando nanoargila em ensaios em batelada com diferentes condições: (I) variação do $\mathrm{pH}$ (7, 8, 9 e 10); (II) variação da massa $(0,02,0,03$ e $0,04 \mathrm{~g})$; (III) variação do tempo de contato (1 e 48 horas). Foi utilizada uma amostra sintética composta de ortofosfato $\left(14 \mathrm{mg} \mathrm{L}^{-1}\right)$, simulando o teor de fósforo encontrado na Represa Billings (SP). Foram realizados bioensaios com sementes de mostarda a fim de avaliar a fitotoxicidade da nanoargila. A remoção de ortofosfato variou de $71 \mathrm{a} 80 \%$, sendo maior com $\mathrm{pH}=8$, massa de $0,04 \mathrm{~g}$ e tempo de contato de 48 horas. Os bioensaios mostraram que a nanoargila não é fitotóxica (IG $=92 \%$ ). Assim, o emprego da nanoargila apresenta cenário promissor na remediação in situ de ambientes aquáticos eutrofizados.
\end{abstract}

Palavras-chave: controle da eutrofização, nanotecnologia, remediação ambiental.

\begin{abstract}
Until 2058 at least 55\% of South America's water bodies will be eutrophic. This phenomenon is triggered by water enrichment by nutrients (Nitrogen and Phosphorus) and results in the excessive production of algae and aquatic plants, reduced dissolved oxygen levels and, as a consequence, increased costs of water treatment. Phosphorus (P), in particular, has been identified as the main element for eutrophication control. Although there are technologies for in situ phosphorus removal (eg. Phoslock ${ }^{\circledR}$ ) its large-scale application is often quite costly. An alternative is nanoclay, which has low cost, is considered inert and has high reactivity with phosphorus. Thus, the present study aimed to evaluate the efficiency of orthophosphate removal using nanoclay in batch assays with different conditions: (I) $\mathrm{pH}$ variation (7, 8, 9 and 10); (II) mass variation (0.02, 0.03 and $0.04 \mathrm{~g}$ ); (III) contact time variation (1 and 48 hours). A synthetic sample composed of orthophosphate (14 mg L $\mathrm{L}^{-1}$ ) was used, simulating the phosphorus content found in the Billings Dam (SP). Mustard seed bioassays were performed to evaluate the nanoclay phytotoxicity. Orthophosphate removal ranged from 71 to $80 \%$, being higher with $\mathrm{pH}=8$, mass of $0.04 \mathrm{~g}$ and contact time of 48 hours. Bioassays showed that nanoclay is not phytotoxic $(G I=92 \%)$. Thus, the use of nanoclay presents a promising scenario for the in situ remediation of eutrophic aquatic environments.
\end{abstract}

Keywords: environmental remediation, eutrophication control, nanotechnology.

\begin{tabular}{|l|l|l|l|l|l|l}
\hline Rev. Bras. Cien., Tec. e Inov. & Uberaba, MG & v. 5 & n. 1 & p. 46-56 & jan./jun. 2020 & ISSN 2359-4748
\end{tabular}




\section{INTRODUÇÃO}

A eutrofização de ecossistemas aquáticos é definida como o aumento da biomassa de organismos fotossintéticos (algas e plantas aquáticas), como consequência do aumento das concentrações de nutrientes. Com isso, a luz do sol não penetra na água e grande parte da biomassa fotossintética morre, de modo que a sua decomposição consome o oxigênio dissolvido na água. Como consequência, ocorre a perda da biodiversidade, perda da qualidade da água e entupimento e degradação de tubulações e bombas, resultando em altos custos para o tratamento da água (SCHINDLER et al., 2016; JEPPESEN; SONDERGAARD; LIU, 2017; VON SPERLING, 2017).

Atualmente, este é um dos principais problemas ambientais que a humanidade enfrenta (SCHINDLER et al., 2016). A fim de contextualizar a realidade regional, em 2010, $41 \%$ dos corpos d'água na América do Sul já enfrentavam problemas de eutrofização (NYENJE et al., 2010). Estima-se que esse número chegue a 55\% até 2058 (WANG et al., 2018). Springer (2017) afirma que o aumento nos níveis de eutrofização pode ocasionar a perda da capacidade de sustentabilidade do sistema, com possibilidades de aumento do nível de toxicidade e comprometimento da saúde humana. Tal situação pode inviabilizar a utilização das águas do manancial para o abastecimento público, indicando que o lançamento de nutrientes no meio ambiente precisa ter maior controle.

O fósforo, em especial, vem sendo apontado como o principal elemento a ser controlado a fim de mitigar o processo de eutrofização em ecossistemas aquáticos (SCHINDLER et al., 2008; SCHINDLER et al., 2016). Como exemplo, no experimento de longa duração realizado por Schindler et al. (2008), constatou-se que, ao enriquecer um lago apenas com fósforo, o sistema se manteve eutrofizado, visto que isto favoreceu 0 desenvolvimento de espécies de cianobactérias fixadoras de nitrogênio atmosférico. Desse modo, como o fósforo não possui fontes atmosféricas, a sua remoção é imprescindível para controlar a eutrofização.

Em contrapartida, toda célula necessita de fósforo simplesmente para sua existência, pois são envolvidas por uma membrana fosfolipídica (GROSS, 2017). Outro aspecto essencial é que o fósforo é constituinte de diversas moléculas importantes para o metabolismo dos seres vivos, como o trifosfato de adenosina (ATP), cuja função é armazenar energia para as atividades vitais básicas das células.

De acordo com Cordell (2017), estima-se que cerca de $80 \%$ do fósforo é perdido na cadeia de abastecimento desde a extração até o uso no setor agrícola, e grande parte acaba em rios e lagos, levando à poluição por nutrientes. Assim, estima-se que as taxas de retorno do fósforo ao seu ciclo biogeoquímico estejam cada vez mais reduzidas em comparação com as suas perdas, com impactos na disponibilidade de suas fontes naturais e na qualidade das águas, em decorrência do processo de eutrofização.

Segundo os estudos de Welch (2009) e Finlay, Small e Sterner (2013), foram comparados dois cenários de remoção: (1) controle de fósforo e (2) controle de fósforo e nitrogênio. Os estudos concluíram que apenas com o controle do fósforo obteve-se a mesma redução do nível de eutrofização. Além disso, Schindler et al. (2016) e Sondergaard et al. (2017) afirmam que, reduzindo a concentração de fósforo, altera-se a relação N/P, o que aumenta a competição de outras espécies com as cianobactérias, e contribui para a redução da eutrofização.

Assim, a fim de restaurar um ambiente aquático eutrofizado, é necessário eliminar as fontes externas de nutrientes, como, por exemplo, através do tratamento adequado dos 
efluentes antes de seu lançamento em corpos d'água. Para isso, existem técnicas de tratamento de efluentes, como a precipitação química (YANG et at., 2013), sistemas de lodos ativados com as devidas modificações (VON SPERLING, 2017), bem como sistemas de alagados construídos (BALDOVI et al., 2021; BENASSI et al., 2018; VYMAZAL, 2007; 2011).

Entretanto, mesmo cessando as fontes externas de nutrientes, o ambiente aquático poderá ser manter eutrofizado por muitos anos, devido às fontes internas de nutrientes. Desse modo, são necessárias medidas de controle do aporte interno de nutrientes, "in situ", como o Phoslock ${ }^{\circledR}$ (LURLING; VAN OOSTERHOUT, 2013; YAMADA-FERRAZ et al., 2015; PHOSLOCK WATER SOLUTIONS, 2017), ou o uso de algicidas para o controle da proliferação de algas. Todavia, o custo associado é elevado e as consequências à comunidade fitoplanctônica com o uso desses produtos pode afetar o equilíbrio do ecossistema aquático (NISHIMURA, 2012). Como exemplo, Pompêo et al. (2013) avaliaram a concentração de diversos metais pesados na água do Reservatório Guarapiranga, localizado na RMSP (Região Metropolitana de São Paulo), e o cobre foi quantificado com valores acima das referências regionais (VRR), associado ao uso intenso de algicidas no local, como o sulfato de cobre.

A nanoargila, por sua vez, é um material de origem natural e que possui propriedades que facilitam a remoção de poluentes da água, tais como: dimensão da ordem de nanômetros, elevada área superficial e carga característica de materiais argilosos (YUAN; WU, 2007). Estudos anteriores apontam o uso de nanoargilas na remoção de diversos poluentes ambientais, tais como: metais pesados, disruptores endócrinos, fármacos, bem como o ortofosfato (YUAN, 2004; YUAN; WU, 2007; RAMANAYAKA et al., 2020).

Desse modo, o presente estudo teve por objetivo avaliar a influência do $\mathrm{pH}$, dosagem e tempo de contato na eficiência de remoção de ortofosfato utilizando nanoargila em ensaios de bancada. Considerou-se tratar de um composto de baixo custo, inerte e que possui alta reatividade com componentes fosforados devido à alta superfície específica. A fim de contextualizar a realidade local, foi simulado o pico de fósforo registrado nas águas da Represa Billings, de $14 \mathrm{mg} \mathrm{L}^{-1}$, objetivando avaliar, de forma preliminar, a possibilidade da utilização da nanoargila como medida de remediação in situ de ambiente aquático eutrofizado. Adicionalmente, foi avaliado o potencial fitotóxico da nanoargila por meio de bioensaios com sementes de mostarda (Sinapis alba).

\section{MATERIAL E MÉTODOS}

Este trabalho baseou-se no estado de eutrofização da Represa Billings, que está localizada a sudeste da RMSP (Figura 1). A oeste faz limite com a Bacia Hidrográfica Guarapiranga e ao sul, com a Serra do Mar (CAPOBIANCO; WHATELY, 2002).

Foi simulado o pico de concentração de ortofosfato ocorrido na Represa Billings em outubro de 2018, com uma concentração de $\mathrm{P}_{-} \mathrm{PO}_{4}{ }^{3-}$ de $14 \mathrm{mg} \mathrm{L}^{-1}$. A Figura 2 ilustra 0 constante bloom de algas nas águas da Represa, registrado em março de 2019. 
Figura 1. Localização da Represa Billings. À esquerda: localização da Represa Billings no Brasil. À direita, em azul, é apresentada a Represa Billings na RMSP

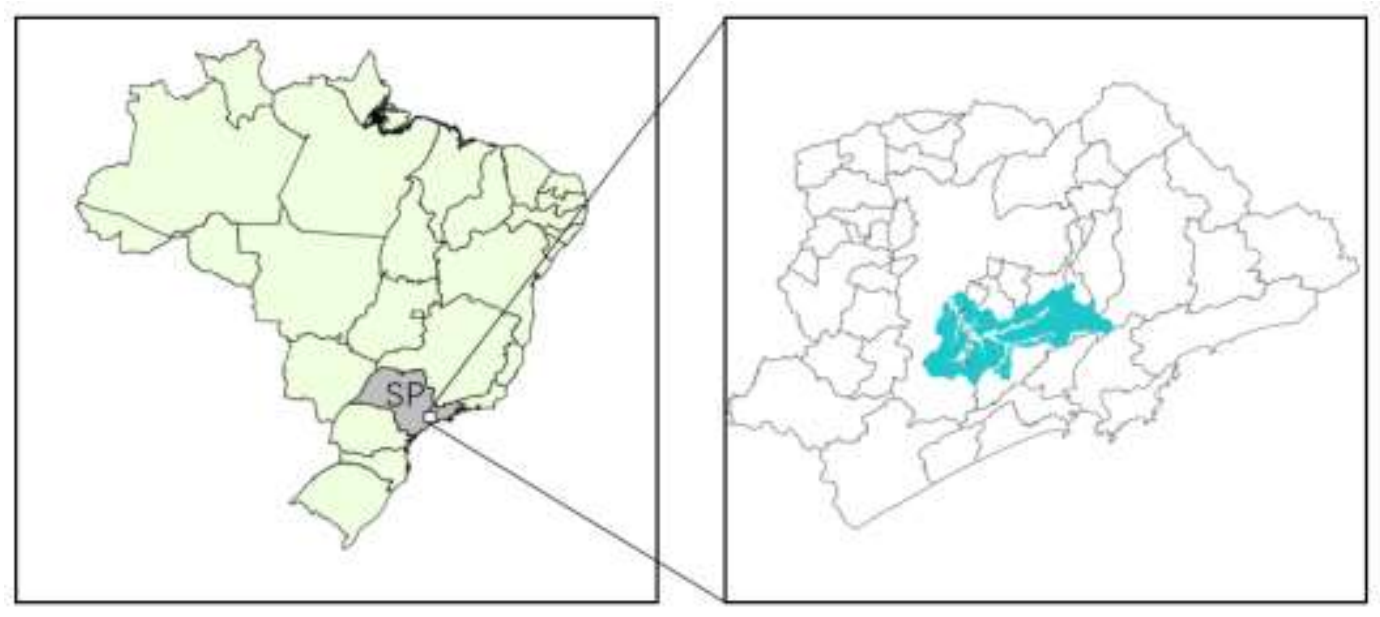

Figura 2. Fotografia da Represa Billings na região de São Bernardo do Campo, SP

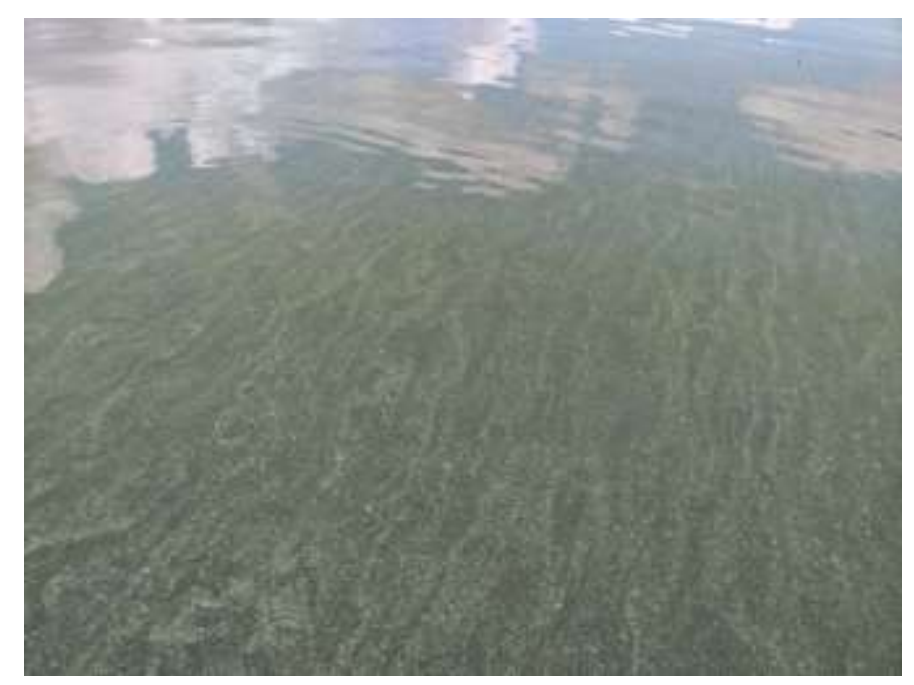

\section{Preparo da solução padrão de ortofosfato}

Para simular tal cenário, foi preparada uma solução padrão de ortofosfato com 14 $\mathrm{mg} \mathrm{L}^{-1}$. O preparo da solução foi realizado mediante a dissolução, em água, de $0,0615 \mathrm{~g}$ de fosfato diácido de potássio $\left(\mathrm{KH}_{2} \mathrm{PO}_{4}\right)$, previamente seco por 1 hora em estufa a $105^{\circ} \mathrm{C}$, e avolumado para 1 litro de água ultrapura.

\section{Ensaios com a nanoargila}

A fim de avaliar a eficiência de remoção de ortofosfato utilizando a nanoargila, foram realizados ensaios em batelada com diferentes condições: (I) variação do $\mathrm{pH}(7,8,9$ e 10); (II) variação da massa $(0,02,0,03$ e $0,04 \mathrm{~g})$ e (III) variação do tempo de contato (1 e 48 horas).

Os ensaios consistiram em adicionar certa massa de nanoargila (ARGILOS, 2018) com $30 \mathrm{~mL}$ da amostra a ser testada em erlenmeyers de $100 \mathrm{~mL}$ e levados para mesa 
agitadora (SOLAB, SL $180 \mathrm{DT})$, por determinado tempo a $100 \mathrm{rpm}$, em temperatura ambiente. Em seguida, as amostras foram filtradas em papel de filtro qualitativo por gravidade, com o objetivo de remover partículas precipitadas na solução. As amostras filtradas foram colocadas em béqueres de $100 \mathrm{~mL}$ para posterior análise das concentrações de ortofosfato. Os ensaios foram realizados em triplicata.

A modificação do $\mathrm{pH}$ das amostras foi realizada utilizando o Titulador Easy Plus da Mettler Toledo, e uma solução $(0,1 \mathrm{M})$ de hidróxido de sódio $(\mathrm{NaOH})$. A massa da nanoargila foi determinada em balança analítica (Shimadzu, ATX 224). Os materiais utilizados nos ensaios foram previamente descontaminados com HCl $(10 \% \mathrm{v} / \mathrm{v})$ e enxaguados com água ultrapura. Amostras controle foram preparadas para posterior comparação da remoção de fosfato pela nanoargila, descontando uma possível remoção ou contaminação no andamento do ensaio.

\section{Análise de ortofosfato}

O preparo da curva de calibração e as análises realizadas nas amostras de água seguiram a metodologia do ácido ascórbico, Método APHA 4500-P E (APHA, 2012). Para a curva de calibração, foram preparadas misturas com concentrações de 6,25 até $500 \mu \mathrm{g}$ $\mathrm{L}^{-1}$. As leituras das absorbâncias foram realizadas em espectrofotômetro (Hach, DR 5000 UV-Vis). Foram analisados também brancos analíticos, para descontar a presença de possíveis interferentes.

Para análise das amostras procedeu-se com a diluição utilizando água deionizada (condutividade elétrica de $0,05 \mu \mathrm{S} \mathrm{cm}^{-1}$ ) visto que as absorbâncias esperadas poderiam ser acima do limite de absorbância do método que é igual a 1. Para valores acima de 1, a curva de calibração não possui mais tendência linear, não sendo possível determinar a concentração de ortofosfato com acurácia.

\section{Bioensaios com sementes de mostarda (Sinapis alba)}

Os bioensaios in vitro com sementes foram realizados conforme metodologia adaptada de Brasil (2009), Belo (2011) e Kohatsu et al. (2018). Os bioensaios consistiram em incubar sementes de mostarda (S. alba) durante 72 horas a $20^{\circ} \mathrm{C}$ em contato com a solução de nanoargila baseados nos resultados da melhor dosagem $\left(1,33 \mathrm{~g} \mathrm{~L}^{-1}\right)$.

As sementes foram acondicionadas em placas de Petri contendo papel filtro qualitativo e $3 \mathrm{~mL}$ da solução de nanoargila $\left(1,33 \mathrm{~g} \mathrm{~L}^{-1}\right)$. Também foi preparado um controle positivo utilizando água ISO, seguindo a metodologia descrita pela OECD (2004), que é uma solução que contém diversos componentes essenciais para o desenvolvimento da radícula. Os bioensaios foram realizados em triplicata.

Após o período de incubação, foi determinado o índice de germinação (IG), que permite classificar as amostras quanto ao seu grau de fitotoxicidade e varia de $I G>100$ (quando a amostra potencializa a germinação) a $I G<30$ (amostra muito fitotóxica). Para obtenção do IG, foi necessário calcular a porcentagem relativa de germinação (\%RSG) e a porcentagem relativa de crescimento radicular (\%RRG), conforme Equações 1 e 2, respectivamente (BELO, 2011):

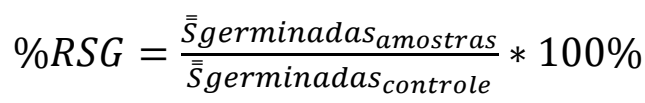


Onde $\overline{\bar{S}}$ germinadas amostras $_{\text {é a média aritmética da quantidade de sementes }}$

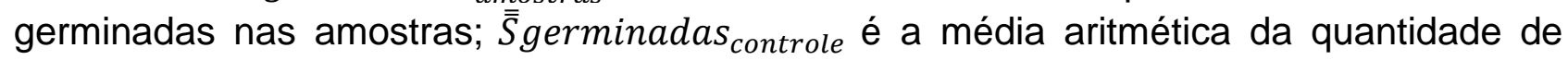
sementes germinadas no controle positivo.

$\% R R G=\frac{\overline{\bar{L}} \text { raízamostras }}{\overline{\bar{L}} \text { raíz } \text { controle }} * 100 \%$

Onde $\overline{\bar{L}}$ raíz $z_{a m o s t r a s}$ é a média aritmética do comprimento das raízes nas amostras; $\bar{L}$ raíz $_{\text {controle }}$ é a média aritmética do comprimento das raízes no controle positivo.

Após determinar \%RSG e \%RRG, foi calculado o Índice de Germinação (IG), através da Equação 3:

$I G=\frac{\% R S G * \% R R G}{100}$

Em seguida, os resultados foram comparados com a escala adaptada de Belo (2011) por Kohatsu et al. (2018), conforme Tabela 1.

Tabela 1. Classificação qualitativa de fitotoxicidade

\begin{tabular}{cc}
\hline IG & Classificação do material em análise \\
\hline$>100$ & O material potencializa a germinação e o crescimento das raízes das plantas \\
$80-100$ & Não fitotóxico, composto maturado \\
$60-80$ & Moderadamente fitotóxico \\
$30-60$ & Fitotóxico \\
$<30$ & Muito fitotóxico \\
\hline
\end{tabular}

Fonte: Adaptado de Belo (2011).

\section{Análise estatística}

Os dados foram analisados estatisticamente por meio de análise de variância (ANOVA) e teste de Tukey, a $5 \%$ de probabilidade. Utilizou-se o programa computacional PAST (HAMMER; HARPER; RYAN, 2001) para essa avaliação.

\section{RESULTADOS E DISCUSSÃO}

Os ensaios com variação de $\mathrm{pH}(7,8,9$ e 10) resultaram em remoções de ortofosfato entre 71 e $77 \%$. Foi observada diferença estatística apenas para o $\mathrm{pH} 10$, onde foi obtida remoção de $71 \%$, que ainda é bastante satisfatória (Figura 3a). Os resultados indicam remoção considerável em uma faixa abrangente de $\mathrm{pH}$, o que é bastante positivo visando a comercialização do produto e a aplicabilidade em ambientes com valores de $\mathrm{pH}$ mais básicos, como na Represa Billings (CETESB, 2016).

O ensaio com variação da dosagem de nanoargila resultou em maior remoção de ortofosfato com o aumento da dosagem de nanoargila aplicada. Assim, a dosagem de 0,02 g para $30 \mathrm{~mL}$ resultou em remoção de $74 \%$ do ortofosfato, já com o aumento da dosagem para $0,04 \mathrm{~g}$ por $30 \mathrm{~mL}$, a remoção aumentou para $80 \%$, com diferença significativa $(\mathrm{p}=$ $0,05)$ (Figura $3 \mathbf{b}$ ). Para o teste com pH 8 e melhor dosagem $(0,04 \mathrm{~g}$ por $30 \mathrm{~mL}$ ) com tempo de contato de 1 hora, foi obtida remoção de apenas 1\% (Figura 3c), denotando que o material precisa de maior tempo de contato para ser efetivo na remediação. 
Yuan e Wu (2007) avaliaram o uso de nanoargila e Phoslock ${ }^{\circledR}$ na remoção de ortofosfato em condições semelhantes às do presente estudo. Os resultados obtidos para a nanoargila foram semelhantes aos do presente estudo ( $70 \%$ de remoção) e superiores aos obtidos pelo Phoslock ${ }^{\circledR}$. Os autores destacam ainda a vantagem da nanoargila apresentar custo cerca de uma ordem de grandeza menor que o do Phoslock ${ }^{\circledR}$.

Já no estudo de Kong et al. (2018), os autores obtiveram elevadas remoções de ortofosfato (quase $100 \%$, partindo de concentração inicial de $100 \mathrm{mg} \mathrm{L}^{-1}$ para ampla faixa de $\mathrm{pH}: 3$ a 11), entretanto, a nanoargila era modificada com hidróxido de lantânio, o que aumentou cerca de 10 vezes a capacidade de remoção em relação à nanoargila sem a modificação. Desse modo, pode-se dizer que nossos resultados são semelhantes aos obtidos por estes autores para a nanoargila sem a modificação.

Figura 3. Ensaios de remoção de ortofosfato pela nanoargila: (a) variação de $\mathrm{pH}$; (b) variação de massa $(\mathrm{g})$; (c) variação do tempo de contato $(\mathrm{h})$

(a)

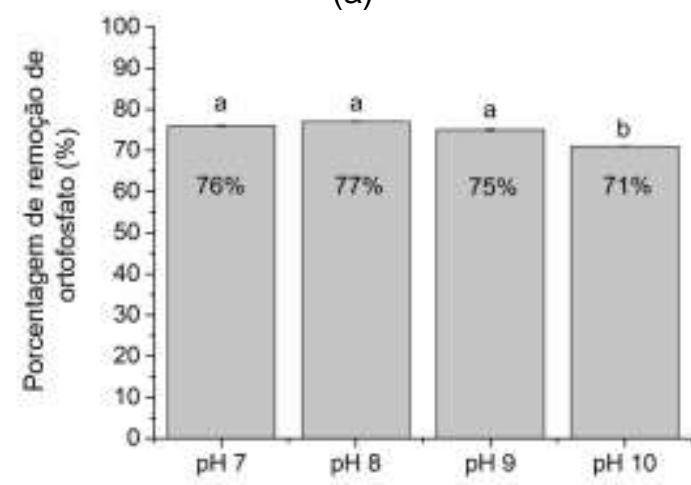

(c)
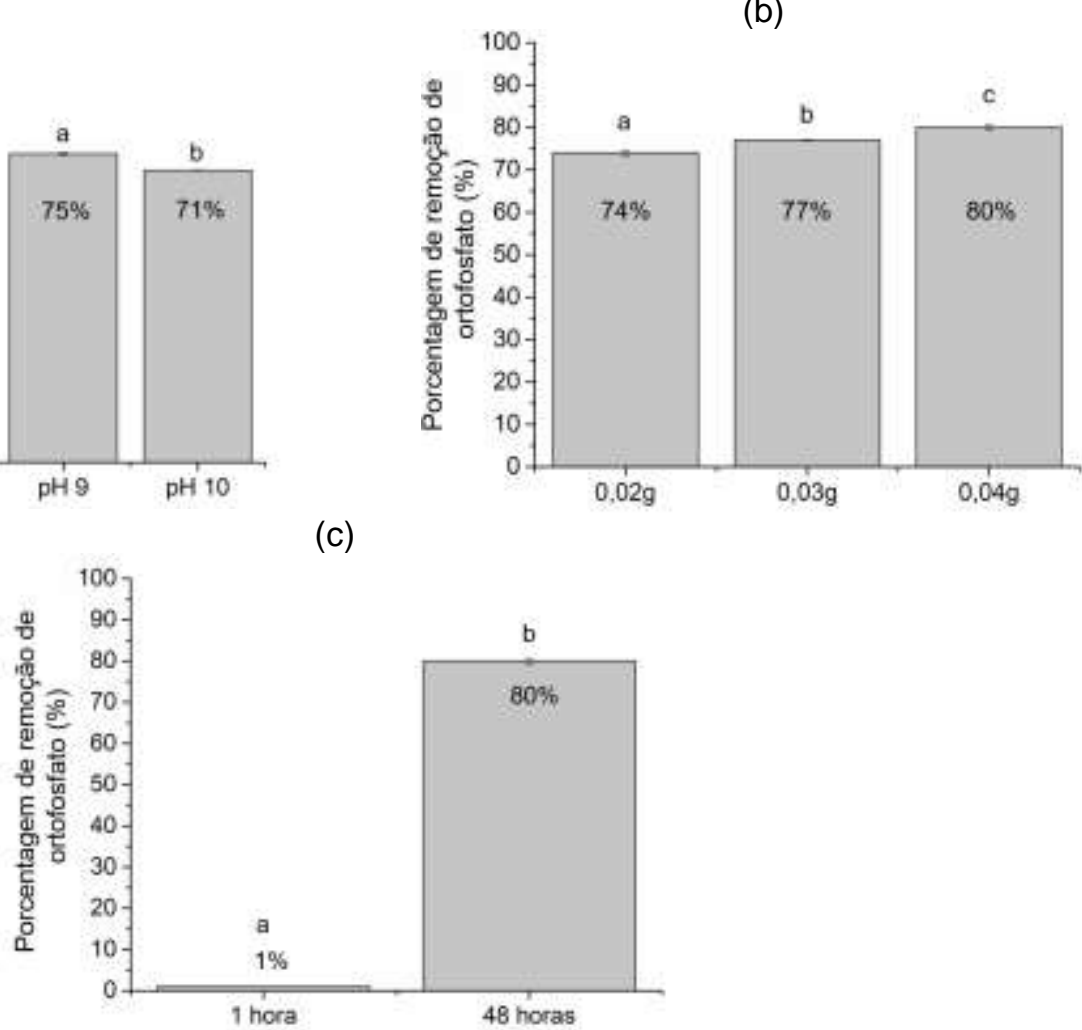

NOTA: As médias seguidas por letras distintas indicam diferença significativa pelo Teste de Tukey.

Outros estudos avaliaram a utilização de outros materiais para a remoção de ortofosfato. Como exemplo, Vieira, Coelho e Jesus (2019) avaliaram a remoção de fosfato utilizando um substrato natural produzido a partir de conchas de marisco moídas. Os resultados obtidos mostraram remoção de $70 \%$ para um tempo de contato de 24 horas com concentração inicial de $0,5 \mathrm{mg} \mathrm{L}^{-1}$. Entretanto, para concentração inicial de $5 \mathrm{mg} \mathrm{L}^{-1} \mathrm{de}$ ortofosfato, a remoção foi de apenas $40 \%$. Assim, são esperadas possíveis variações de remoção de ortofosfato da coluna d'água de ambiente a ser remediado com a nanoargila. Entretanto, como no presente estudo trabalhamos com o maior valor registrado para a realidade local (14 $\left.\mathrm{mg} \mathrm{L}^{-1}\right)$, as remoções, provavelmente poderiam ser ainda maiores que 
os $80 \%$ obtidos, ou poder-se-ia aplicar menores dosagens em locais cujos teores de fósforo fossem menores, mantendo a mesma eficiência.

Os resultados dos testes de fitotoxicidade mostraram que o \%RRG foi de $92 \%$. Já o \%RSG foi de $100 \%$. Portanto, a nanoargila não foi fitotóxica (IG = 92\%), conforme Tabela 1 de Belo (2011). Isso implica que o uso da nanoargila não afetou o desenvolvimento da espécie vegetal ( $S$. alba). Em comparação com os resultados encontrados na literatura e considerando a Tabela 1 como classificação da fitotoxicidade, Kohatsu et al. (2018) classificaram a água de um córrego que recebe despejos de esgoto bruto (Córrego Ribeirão Pires, SP) como moderadamente fitotóxica ( $\mathrm{G}=74 \%$ ). Ressalta-se que o bioensaio com S. alba é bastante sensível e que também consegue detectar a fitotoxicidade mesmo quando o poluente está em baixas concentrações. Como exemplo, Vokřál et al. (2019) testaram a fitotoxicidade da Ivermectina, um medicamento anti-helmíntico de uso veterinário e humano. Foram avaliadas concentrações de 50 e $500 \mathrm{nM}$ e um controle sem a droga. Os autores observaram diferença significativa entre o crescimento das raízes das sementes de $S$. alba em relação ao controle, com diminuição do crescimento em cerca de $20 \%$ nos bioensaios contendo a Ivermectina, demonstrando a toxicidade da droga para a espécie vegetal, mesmo em baixa concentração.

Desse modo, os resultados obtidos demonstram o potencial da nanoargila como ecotecnologia de baixo custo para a remoção de ortofosfato da água em ampla faixa de $\mathrm{pH}$ (7 a 10). Além disso, os bioensaios indicaram que a nanoargila não é fitotóxica, sendo mais um ponto favorável à sua aplicação na remediação de ambiente aquático, visto que não apresentará riscos de inserção de substância tóxica no ecossistema. Assim, sua aplicação na remediação in situ de ecossistemas aquáticos eutrofizados é bastante promissora. Os resultados também permitem inferir que a nanoargila poderia também ser aplicada para o tratamento terciário de efluentes e evitar que o ortofosfato chegue aos corpos d'água e, assim, evitar a eutrofização dos corpos d'água. Nesse cenário, poder-se-ia, inclusive, recuperar o fósforo adsorvido à nanoargila por sedimentação e reaproveitá-lo na agricultura (YUAN; WU, 2007). O reaproveitamento do fósforo para uso agrícola é uma preocupação mundial atual, dado que as reservas naturais estão se esgotando (CORDELL, 2017).

\section{CONCLUSÕES}

A nanoargila analisada é uma tecnologia de produção local, abundante, inerte e de alta reatividade. Os resultados apresentados mostraram que para dosagem de 0,03 g para $30 \mathrm{~mL}$ e todos os valores de $\mathrm{pH}$ analisados $(7,8,9$ e 10), a remoção de ortofosfato foi acima de $70 \%$, sendo a concentração inicial de $14 \mathrm{mg} \mathrm{L}^{-1}$. Além disso, com o aumento da dosagem $(0,04 \mathrm{~g})$, a remoção atingiu o valor de $80 \%$. Outro fato importante foi que a nanoargila não foi fitotóxica para as sementes de mostarda, mostrando que, provavelmente, este material não é tóxico para as espécies vegetais.

Considerando os resultados obtidos, o uso de nanoargila para a remoção de fósforo apresenta um cenário promissor onde a nanoargila pode, no futuro, ser utilizada em escala comercial, visando a mitigação do processo de eutrofização em ecossistemas aquáticos. Assim, é mais um possível nanomaterial para a remoção de fósforo da água.

Ressalta-se a necessidade de testes em maior escala, como os desenvolvidos por Yamada-Ferraz et al. (2015), a fim de avaliar a aplicação em cenários mais próximos da realidade antes de sua aplicação em larga escala, bem como de seus efeitos em longo prazo. 


\section{REFERÊNCIAS}

APHA. American Public Health Association. Standard Methods for the examination of water and wastewater. Método 4500 P.E. - Método do ácido ascórbico. Washington, 2012.

ARGILOS. Eco-friendly specialty minerals for Mining Solutions. Proposta: Pitch Sabesp, 2018. $15 \mathrm{p}$.

BALDOVI, A. A.; AGUIAR, A. R. B.; BENASSI, R. F.; VYMAZAL, J.; JESUS, T. A. Phosphorus removal in a pilot scale free water surface constructed wetland: hydraulic retention time... Chemosphere, v. 266, p. 128939, 2021.

BELO, S. R. Avaliação de fitotoxicidade através de Lepidium sativum no âmbito de processos de compostagem. 2011. 79 f. Dissertação (Mestrado em Engenharia Mecânica) - Universidade de Coimbra, Portugal, 2011.

BENASSI, R. F.; MATHEUS, D. R.; SUBTIL, E. L.; COELHO, L. H. G.; OLIVEIRA, L. H. S.; MORETTO, M. R. D. et al. Manual de sistemas de wetlands construídas para 0 tratamento de esgotos sanitários. Santo André: Editora Copiart, 2018. 55 p.

BRASIL. Ministério da Agricultura, Pecuária e Abastecimento. Regras para análises de sementes. Secretaria de Defesa Agropecuária. Brasília: Mapa/ACS, 2009. 399 p.

CAPOBIANCO, R. P. J; WHATELY, M. Billings 2000: ameaças e perspectivas para o maior reservatório de água da região metropolitana de São Paulo. São Paulo: Instituto Socioambiental de São Paulo, 2002. 59 p.

CETESB. Companhia Ambiental do Estado de São Paulo. Relatório da qualidade das águas interiores no estado de São Paulo em 2016. Disponível em: http://cetesb.sp.gov.br/aguas-interiores/wpcontent/uploads/sites/12/2013/11/Cetesb_QualidadeAguasInteriores_2016_corre\%C3\%A 7\%C3\%A3o02-11.pdf. Acesso em: 13 de janeiro de 2019.

CORDELL, D. Global Phosphorus scarcity: A food secure future? Journal of Nutrition \& Intermediary Metabolism, v. 8, p. 61-62, 2017.

FINLAY, J. C.; SMALL, G. E.; STERNER, R. W. Human influences on nitrogen removal in lakes. Science, v. 342, n. 6155, p. 247-250, 2013.

GROSS, M. Where is all the phosphorus? Current Biology, v. 27, n. 21, p. 1141-1155, 2017.

HAMMER, $\varnothing$.; HARPER, D. A. T.; RYAN, P. D. PAST: Paleontological statistics software package for education and data analysis. Palaeontologia Electronica, v. 4, n. 1, p. 1-9, 2001.

JEPPESEN, E.; SONDERGAARD, M.; LIU, Z. Lake Restoration and Management in a Climate Change Perspective: An Introduction. Water, v. 9, n. 2, 2017. 
KOHATSU, M. Y.; JESUS, T. A.; COELHO, L. H. G.; PEIXOTO, D. C.; POCCIA, G. T.; HUNTER, C. Fitotoxicidade de água superficial da Região Metropolitana de São Paulo utilizando bioensaio com Sinapis alba. Acta Brasiliensis, v. 2, n. 2, p. 58-62, 2018.

KONG, L.; TIAN, Y.; LI, N.; LIU, Y.; ZHANG, J.; ZHANG, J. et al. Highly-effective phosphate removal from aqueous solutions by calcined nano-porous palygorskite matrix with embedded lanthanum hydroxide. Applied Clay Science, v. 162, p. 507-517, 2018.

LURLING, M.; VAN OOSTERHOUT, F. Controlling eutrophication by combined bloom precipitation and sediment phosphorus inactivation. Water Research, v. 47, p. 6527-6537, 2013.

NISHIMURA, P. Y. A comunidade fitoplanctônica nas Represas Billings e Guarapiranga (Região Metropolitana de São Paulo). 2012. 149 f. Tese (Doutorado em Ecologia). Instituto de Biociências. Universidade de São Paulo, 2012.

NYENJE P. M.; FOPPEN J. W.; UHLENBROOK S.; KULABAKO R.; MUWANGA A. Eutrophication and nutrient release in urban areas of sub-Saharan Africa - A review. Science of the Total Environment, v. 408, n. 3, p. 447-455, 2010.

OECD. Organização para a Cooperação e Desenvolvimento Econômico. Test n. 202: Daphnia sp. Acute Immobilisation Test, OECD Guidelines for the Testing of Chemicals, Section 2. Paris: OECD Publishing, 2004. Disponível em: \&lt;https://doi.org/10.1787/9789264069947-en\&gt. Acesso em: 10 jul. 2019.

PHOSLOCK WATER SOLUTIONS. Recuperação da Lagoa da Pampulha, Belo Horizonte (SUDECAP/PBH). Disponível em: http://phoslock.com.br/casos-deuso/recuperacao-da-lagoa-da-pampulha-belo-horizonte-sudecappbh/. Acesso em: 11 mar. 2019.

POMPÊO, M.; PADIAL, P. R.; MARIANI, C. F.; CARDOSO-SILVA, S.; MOSCHINICARLOS, V.; SILVA, D. C. V. R. et al. Biodisponibilidade de metais no sedimento de um reservatório tropical urbano (reservatório Guarapiranga - São Paulo (SP), Brasil). Geochimica Brasiliensis, v. 27, n. 2, p. 104-119, 2013.

RAMANAYAKA, S.; SARKAR, B.; COORAY, A.T.; OK, Y.S. Halloysite nanoclay supported adsorptive removal of oxytetracycline antibiotic from aqueous media. Journal of Hazardous Materials, v. 384, p. 121301, 2020.

SCHINDLER, D. W. S. R.; CARPENTER, S. C.; CHAPRA, R. E.; HECKY, D. M.; ORIHEL. Reducing phosphorus to curb lake eutrophication is a success. Environmental Science \& Technology, v. 50, n.17, p. 8923-8929, 2016.

SCHINDLER, D. W.; HECKY, R. E.; FINDLAY, D. L.; STAINTON, M. P.; PARKER, B. R.; PATERSON, M. J. et al. Eutrophication of lakes cannot be controlled by reducing nitrogen inputs: Results of a 37-years whole-ecosystem experiment. PNAS, v. 105, n. 32, p. 1125411258, 2008. 
SONDERGAARD, M.; LAURIDSEN, T., L.; JOHANSON, L. S.; JEPPESEN, E. Nitrogen or phosphorus limitation in lakes and its impact on phytoplankton biomass and submerged macrophyte cover. Hydrobiologia, v. 795, n. 1, p. 35-48, 2017.

SPRINGER, N. P. Physical, technical, and economic accessibility of resources and reserves need to be distinguished by grade: Application to the case of phosphorus. Science of the Total Environment, v. 577, p. 319-328, 2017.

VIEIRA, B.; COELHO, L H. G.; JESUS, T. A. Phosphate Sorption in Shellfish Shell (Venerupis pulastra) Substrates: Development of Green and Low-Cost Technology for Tertiary Treatment of Effluents. Journal of Environmental Engineering, v.145, n. 2, 2019.

VOKŘÁL, I.; MICHAELA, S.; RADKA, P.; JIŘÍ, L.; LUKÁŠ, P.; DOMINIKA, S. et al. Ivermectin environmental impact: Excretion profile in sheep and phytotoxic effect in Sinapis alba. Ecotoxicology and Environmental Safety, v. 169, p. 944-949, 2019.

VON SPERLING, M. Introdução à qualidade das águas e ao tratamento de esgotos. v. 1. 4. ed. Belo Horizonte: Universidade Federal de Minas Gerais, 2017. 452 p.

VYMAZAL, J. Removal of nutrients in various types of constructed wetlands. Science of the Total Environment, v. 380, p. 48-65, 2007.

VYMAZAL, J. Constructed wetlands for wastewater treatment: five decades of experience. Environmental Science and Technology, v. 45, p. 61-69, 2011.

WANG, S.; LI, J.; ZHANG, B.; SPYRAKOS, E.; SHEN, Q.; LEHMANN, K. et al. Trophic state assessment of global inland waters using a MODIS-derived Forel-Ule index. Remote Sensing of Environment, v. 217, p. 444-460, 2018.

WELCH, E. B. Should nitrogen be reduced to manage eutrophication if it is growth limiting? Evidence from Moses Lake. Lake Reservoir Manage, v. 25, n. 4, p. 401-409, 2009.

YAMADA-FERRAZ, T. M.; SUEITT, A. P. E.; OLIVEIRA, A. F.; BOTTA, C. M. R.; FADINI, P. S.; NASCIMENTO, M. R. L. et al. Assessment of Phoslock application in a tropical eutrophic reservoir: An integrated evaluation from laboratory to field experiments. Environmental Technology \& Innovation, v. 4, p. 194-205, 2015.

YANG, S.; DING, D.; ZHAO, Y.; HUANG, W.; ZHANG, Z.; LEI, Z. et al. Investigation of phosphate adsorption from aqueous solution using Kanuma mud: Behaviors and mechanisms. Journal of Environmental Chemical Engineering, v. 1, n. 3, p. 355-362, 2013.

YUAN, G. Natural and modified nanomaterials as sorbents of environmental contaminants, Journal of Environmental Science Health, part A, v. 39, p. 2661-2670, 2004.

YUAN, G.; WU, L. Allophane nanoclay for the removal of phosphorus in water and wastewater. Science and Technology of Advanced Materials, v. 8, p. 60-62, 2007. 